
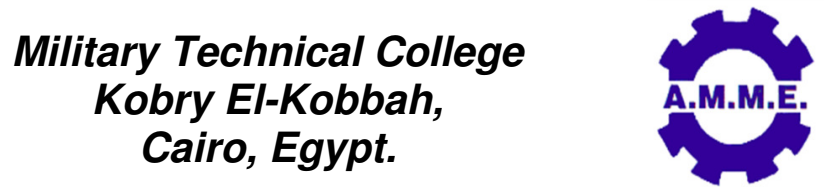

$18^{\text {th }}$ International Conference on Applied Mechanics and Mechanical Engineering.

\title{
EFFECT OF TOLERANCES OF SMALL SIZE SLIDER-CRANK MECHANISM ON POSITION ERRORS AND UNCERTAINTY
}

\author{
E. Rashed ${ }^{1}$, M. Damir ${ }^{2}$ and A. Elkhatib ${ }^{2}$
}

\begin{abstract}
Small size slider-crank mechanisms are used in many accurate and precise sophisticated applications. The design parameters of the mechanism play an important role in defining its performance characteristics. The input-output relations are the main criteria to define its characteristics and hence its capabilities to achieve specific application requirement. The tolerance analysis for mechanism components are a key element for studying and improving mechanism characteristics and its transmission quality. In this paper kinematics models for small size slider-crank mechanism are presented. The models consider the mechanism characteristic range, sensitivity and input-output relations. The effect of mechanism manufacturing and assembly errors such as; components tolerances, joints clearances and assembly deviations were investigated. The study elaborates the effect of these errors on the mechanism characteristic and position uncertainty. A contact approach model is introduced analyzing the effect of joints clearances on mechanism hysteresis error, position uncertainty, and dead zones. The study is further condensed to define the most appropriate design parameters needed for different applications.
\end{abstract}

\section{KEY WORDS}

Slider-crank mechanism, Kinematic analysis, Dimensional assembly tolerance, System sensitivity and accuracy, Revolute joint clearances.

Assistant lecturer, Production Engineering Dept., Faculty of Engineering, Alexandria University, Alexandria, Egypt. (E-mail: enjy.osama@alexu.edu.eg).

2 Professors, Production Engineering Dept., Faculty of Engineering, Alexandria University, Alex., Egypt. (E-mail: mohamed.damir@alexu.edu.eg)(E-mail: elkhatib40@alexu.edu.eg). 


\section{INTRODUCTION}

The slider crank mechanism is widely used in many engineering and medical applications. The mechanism is used as a motion transmission system to convert rotating motion into linear motion and vice versa.

Large scale mechanisms have wide applications in reciprocating engines and machine tools where the dynamics of the systems are its main criteria. Small size mechanisms are very significant in many aspects where very accurate and precise movements are required such as measuring instruments, micro electro mechanical systems (MEMS), biomimetics and endoscopic surgical tools [1, 2].

The study of the performance of the slider crank mechanism found great interest for many investigators. Most of the researches investigate the effect of the mechanism design parameters on the system dynamic behavior [3,4,11]. Lee and Gilmore [5] studied the effects of the tolerance in the link length, joint clearance on the performance of the mechanisms.

Some researchers analyzed the kinematic of the mechanism taking into consideration joint clearance [6,7]. Choi and Lee [8] simulate the joint clearance in four bar and slider crank mechanism based on the clearance vector concept. Venanzi [9] developed a new method to study the effect of misalignment in revolute, prismatic, cylindrical, and spherical joints on the performance of the mechanism. Flores et al. [10] studied the dimensional tolerance effect on the position accuracy in slider crank mechanism. Ting et al. [11] developed a kinematic model to study the effect of joint clearances in the mechanism performance using rotatability law.

Many investigators analyzed the kinematic error in the micro and macro mechanisms systems applied for robotics and mechatronics [12-14].

In the present work, for small scale mechanisms, analytical kinematics studies are introduced. The study considers the role of the basic design parameters on the mechanism characteristics and sensitivity. The effect of mechanism manufacturing and assembly errors namely; components tolerances, joints clearances and assembly deviations were investigated. The resultant errors and uncertainties in mechanism output due to mechanism elements tolerances and assembly errors are studied. A new approach, based on continuous contact mode is introduced to elaborate the effect of revolute joints clearances on the mechanism performance and uncertainty.

\section{KINEMATIC MODELING}

Slider-crank mechanism is widely used in many applications where transformation from rotational into translational motion (or vice versa) is required. The use of this mechanism is common in many engineering applications such as robotics and mechatronic systems where complex motions are to be with rotational actuators.

Small scale slider crank mechanism has also many applications where, high precision and accuracy are required, such as magnification systems and control in measuring instruments. Beside it has wide applications in endoscopy surgical tools, the slider mechanism is mainly composed of a rotating driving link (crank), a follower 
link (connecting rod) and a sliding body (slider), with three revolute and one prismatic joints.

There are two main types of slider crank mechanism; the inline and the offset types, where the pivot point of the crank is either coincident or shifted with respect to the axis of the slider linear motion as shown in Figs. 1 and 2.

The main design parameters of the mechanism are; the center distance between the links joints holes, i.e. the crank length (a) and connecting rod length (b). The choices of these dimensions not only define the mechanism path length and mechanism input- output relations but also affect their function quality. The kinematic analysis of the mechanism is the key for their design, and for studying their performance and hence evaluating their transmission quality.

For small scale application of the mechanism, where a limited space in the system designs is available. The mechanism is not only used to transfer motion within specific range but also to achieve the required sensitivity and to attain certain degree of accuracy and precision.

The present analysis is aimed to model these constraints. The kinematic equation governing the input-output relation for the case of inline slider crank mechanism can be expressed for different input cases as follows:

I) When the rotation of the crank $(\theta 1)$ drives the linear motion of the slider $(X)$

$$
\begin{aligned}
& X=(a \cos \theta 1)+\sqrt{b^{2}-(a \sin \theta 1)^{2}}, \text { for }(a \leq b) \text { and }\left(a>b \text { from } \theta 1=0 \text { to } \theta_{\text {max. }}\right) \\
& X=(a \cos \theta 1)-\sqrt{b^{2}-(a \sin \theta 1)^{2}}, \quad \text { for }\left(a>b \text { from } \theta 1=\theta_{\text {max. }} \text { to } 0\right)
\end{aligned}
$$

$\theta 1_{\max }$ is the angle where singularity occurs

$$
\theta 1(\max .)=\sin ^{-1} \frac{b}{a}
$$

\section{II) When the movement of the slider $(\mathrm{X})$ drives the rotation motion of the} $\operatorname{crank}(\theta 1)$

$$
\theta 1=\cos ^{-1}\left(\frac{a^{2}-b^{2}+(X)^{2}}{2 a(X)}\right) \quad, \quad \text { for }(a \leq b) \text { and }(a>b)
$$

The above relations state the role of the basic design parameters ( $a$ and $b$ ) on the mechanism characteristics. The main design criteria in small scale mechanism are to achieve the required range, which define the maximum and minimum input motion, at appropriate system sensitivity. The system sensitivities (S), as the rate of change of system output with respect to its input, are concluded to be as follows:

i) For input crank rotation, for $(a \leq b)$ and $(a>b$ from $\theta 1=0$ to $\theta$ max.) 


$$
\frac{\partial X}{\partial \theta 1}=(-a \sin \theta 1)+\frac{\left(-a^{2} \sin \theta 1 \cos \theta 1\right)}{\sqrt{b^{2}-(a \sin \theta 1)^{2}}}
$$

ii) For input crank rotation, for ( $a>b$ from $\theta 1=\theta_{\max }$ to 0 )

$$
\frac{\partial X}{\partial \theta 1}=\left(-\mathrm{a} \sin \theta_{1}\right)+\frac{\left(a^{2} \sin \theta_{1} \cos \theta_{1}\right)}{\sqrt{b-\left(a \sin \theta_{1}\right)^{2}}}
$$

iii) For input slider travel, for equation (4)

$$
\frac{\partial \theta 1}{\partial X}=\frac{a^{2}-X^{2}-b^{2}}{2 a X^{2} \times \sqrt{1-\left(\frac{a^{2}+X^{2}-b^{2}}{2 a X}\right)^{2}}}
$$

Through these work, three different cases; $a=b, a>b$ and $a<b$, with $a+b=16 \mathrm{~mm}$, were considered to elaborate the effect of basic design parameter (equations 1 - 6) on the mechanism range and sensitivity. These are carried out for the range of rotational angle $\theta 1=0$ to 90 degrees. The relations illustrate how both the range and sensitivity are varied for each of the cases as shown in Figs. 3 and 4 . These relations are helpful in defining the basic dimension for optimum range of application and required sensitivity.

Using Figs. 3, 4 and 5, the designer could choose the suitable design parameter (a and b) according to the input range of application (e.g. $\Delta \theta$ ). To attain the suitable corresponding sensitivity. In case of $a>b$, as illustrated in Fig.3, the designer could use $\Delta \theta$ either counter clockwise or clockwise according to the corresponding sensitivity required $(\Delta \mathrm{X} / \Delta \theta)$. For the same range $\Delta \theta$ e.g. $(\Delta \mathrm{X} / \Delta \theta)$. For c.c.w. is greater than $(\Delta \mathrm{X} / \Delta \theta)$ for C.w.

\section{ASSEMBLY TOLERANCES ANALYSIS}

The assembly tolerances analysis for mechanism components are a key element for studying and improving mechanism characteristics and quality. There are three main sources of variation in mechanical assembly: 1) Dimensional tolerance.

2) Geometrical tolerance, 3) Deviation due to small kinematic adjustments.

The effect of the mechanism manufacturing and assembly errors; namely components dimensional tolerances $(T)$, joints clearance $(\delta)$ and deviation between slider travel axis and the crank rotating pivot, [offset (e)] on the mechanism accuracy and uncertainty are investigated.

\section{Component Dimensional Tolerances}

Due to the unavoidable manufacturing errors, tolerances on links dimensions are expected, which represent the six-sigma variation in the basic size of the dimensions. These tolerances in mechanism elements will affect the uncertainty of mechanism performance. 
The effect of these tolerances on the uncertainty of the function could be determined by applying the statistical model of propagation of error on the mechanism functions relations (equations 1 and 2).

$$
6 \sigma_{x}=\sqrt{\sum \mathrm{S}_{i}^{2} \mathrm{~T}_{i}^{2}}
$$

where; $\left(\sigma_{x}\right)$ is the function uncertainty, $\left(T_{i}\right)$ is the tolerance value of the mechanism link, $\sigma_{a}=\left(T_{a} / 6\right), \sigma_{b}=\left(T_{b} / 6\right)$; and $\left(S_{i}\right)^{2}$ is the Link tolerance sensitivity index for the element (i).

By Appling propagation of error for equations (1 or 2), the mechanism output uncertainty $(\sigma)$ is as follows;

$$
\sigma_{x}=\sqrt{\left[\left(\frac{\partial X}{\partial a}\right)^{2}(\sigma a)^{2}\right]+\left[\left(\frac{\partial X}{\partial b}\right)^{2}(\sigma b)^{2}\right]}
$$

or

$$
\sigma_{\theta 1}=\sqrt{\left[\left(\frac{\partial \theta 1}{\partial a}\right)^{2}(\sigma a)^{2}\right]+\left[\left(\frac{\partial \theta 1}{\partial b}\right)^{2}(\sigma b)^{2}\right]}
$$

To achieve accurate and precise output, and hence high transmission quality, minimum value of output uncertainty aimed to be attained. This could be achieved through optimum minimization for both $S_{i}$ and $T_{i}$ of the mechanism elements.

Tolerance sensitivity indices $\left(\mathrm{S}_{\mathrm{i}}\right)$ are the measure for the effect of dimension of the mechanism elements on the output function uncertainty. These tolerance sensitivity indices for each link are given by, i.e.:

For the crank,

For the connecting rod

$$
\left(\frac{\partial X}{\partial a}\right)^{2}=\left[(\cos \theta 1)+\frac{-\left(a(\sin \theta 1)^{2}\right)}{\sqrt{b^{2}-(a \sin \theta 1)^{2}}}\right]^{2}
$$

$$
\left(\frac{\partial X}{\partial b}\right)^{2}=\left[\frac{b}{\sqrt{b^{2}-(a \sin \theta 1)^{2}}}\right]^{2}
$$

The relations between the sensitivity indices and the input crank angle for the three studied cases are shown in Figs. 5 ( $\mathrm{i}$ and ii).

\section{Deviation due to Assembly Errors (Offset Error)}

Due to the unavoidable assembly error, a shift may occur between the sliding direction and the crank pivot. This will result in a case of offset slider crank mechanism because the constrained path of the slider does not extend through the center of the crank rotation as shown in Fig. 2. 
This offset error (e) is the distance between the crank pivot and the sliding axis. With the presence of an offset, the performance characteristic of the mechanism will differ. The offset could be considered positive or negative with reference to the deviation between the direction of sliding motion and the crank pivot center line (Fig.2).

The kinematic relation for the mechanism will be:

i) For positive offset case (Fig. 2(i)): the kinematic equation is

$$
X=(a \cos \theta 1)+\sqrt{b^{2}-((a \sin \theta 1)-e)^{2}}
$$

ii) For negative offset case (Fig. 2(ii)): the kinematic equation is

$$
X=(a \cos \theta 1)+\sqrt{b^{2}-((a \sin \theta 1)+e)^{2}}
$$

Figure 6 shows the input-output relations for crank mechanism with positive and negative offset as compared with inline case. From the above relations, it is seen that, due to the offset error (e) a deviation (E) in the mechanism characteristics is resulted.

To evaluate such errors $(E)$ the variation in the input-output relations for the cases of offset and inline conditions are compared.

$$
E=X_{(\text {offset })}-X_{(\text {inline })}
$$

For case of positive offset:

$$
E=\sqrt{b^{2}-((a \sin \theta 1)-e)^{2}}-\sqrt{b^{2}-(a \sin \theta 1)^{2}}
$$

For case of negative offset:

$$
E=\sqrt{b^{2}-((a \sin \theta 1)+e)^{2}}-\sqrt{b^{2}-(a \sin \theta 1)^{2}}
$$

As shown in Fig. 7 the errors $(E)$ are varied with the input angles, and the permissible offset error (e) could be determined according to the allowable permissible error (E).

\section{Mechanism Joints Clearances}

The existence of clearances in the joints of mechanical systems is inevitable due to assemblage and manufacturing errors. An appropriate clearance for joints between links of a mechanical system is necessary to allow for the relative motion of the connected links, as well as to permit assembly of the mechanical system.

Providing that the pin is in continues contact with the hole of the joint. Thus, the joint clearance $\delta$ can be regarded as a short link which is considered a virtual, mass less link whose length is equal to one half of the joint diametric clearance (called a clearance link). 


\section{PROPOSED CONTACT APPROACH MODEL}

In modeling the slider crank mechanism, with revolute joint clearance, the continuous contact mode in which contact between the links joint is assumed to be always maintained. Neglecting the friction in the joint, then the direction of the clearance vector will coincide with the normal direction of the collision plane.

The approach is applied in the cases of the existence of the clearance between the crank and the connecting rod joint $\left(\delta_{C}\right)$, and for the joint between the slider and connecting rod $\left(\delta_{S}\right)$.

The presented contact approach model simulates the input-output function relation of the mechanism. For counter clock wise motion at start, for input angle $(\theta 1)$ equal to zero, and due to the crank clearance $\left(\delta_{C}\right)$, the crank will rotate an angle $\left(\theta_{R}\right)$ without transmitting motion to the connecting rod as shown in Fig. 8 . This angle presented by $\left(\theta_{R}\right)(A-B$ zone) as shown in Fig. 9 can be considered as a system dead zone and is given by:

$$
\theta_{R}=\cos ^{-1} \frac{a^{2}+\left(X_{i}-b\right)^{2}-\left(\delta_{C}\right)^{2}}{2 a\left(X_{i}-b\right)}
$$

where; $X_{i}=(a+b)$. As the crank moves further from $\theta_{R}$ till $\theta 1=90$, then the mechanism functioning as illustrated by ( $\mathrm{B}-\mathrm{C}$ zone), and hence the input-output relation can be presented by:

$$
X_{1}=(a \cos \theta 1)+\left(\delta_{C} \sin \theta 1\right)+\sqrt{b^{2}-\left((a \sin \theta 1)-\left(\delta_{C} \cos \theta 1\right)\right)^{2}}
$$

To study the uncertainty of the system, the mechanism is considered to move back from $\theta 1=90$ degrees in clock wise direction, similarly, due to the crank diametric clearance $\left(2 \delta_{c}\right)$, it will rotate to angle $\theta_{s}$ without transferring the motion to the slider ( $C$ - $\mathrm{D}$, return dead zone), after which the slider begins to move.

$$
\theta_{S}=90-\left(2 \times \tan ^{-1} \frac{\delta_{C}}{a}\right)
$$

For the crank rotation from angle $\theta_{S}$ to angle $\theta 1=$ zero, the input-output relation is presented by:

$$
X_{2}=\left[(a \cos \theta 1)-\left(\delta_{C} \sin \theta 1\right)\right]+\sqrt{b^{2}-\left((a \sin \theta 1)+\left(\delta_{C} \cos \theta 1\right)\right)^{2}}
$$

As shown in Fig. 9, the existence of joint clearance will result in system inaccuracy (dead zone) and hysteresis error.

The system hysteresis error $\left(\mathrm{H}_{\mathrm{c}}\right)$, as the difference between the counterclockwise (equation 17) and clockwise movements (equation 19), the input-output relation is:

$$
\begin{gathered}
H_{C}=\left[\sqrt{b^{2}-\left((a \sin \theta 1)-\left(\delta_{C} \cos \theta 1\right)\right)^{2}}-\sqrt{b^{2}-\left((a \sin \theta 1)+\left(\delta_{C} \cos \theta 1\right)\right)^{2}}\right] \\
+\left[2\left(\delta_{C} \sin \theta 1\right)\right]
\end{gathered}
$$

Hence, for the six sigma limits, the system position uncertainty $\left(C_{c}\right)$, will be $C_{c}=H_{c} / 6$, 
Similarly, as the contact approach method applied for the case of the slider joint clearance as shown in Fig. 10, the hysteresis error $\left(\mathrm{H}_{\mathrm{s}}\right)$, and hence the system position uncertainty $\left(\mathrm{C}_{\mathrm{s}}\right)$ be,

$$
H_{S}=6 C_{S}=\sqrt{\left(\left(b+\delta_{S}\right)^{2}-(a \sin \theta 1)\right)^{2}}-\sqrt{\left(\left(b-\delta_{S}\right)^{2}-(a \sin \theta 1)\right)^{2}}
$$

The proposed contact method elaborated the effect of revolute joint clearance on the mechanism output performance. Thus, the expected resultant errors are achieved.

\section{CONCLUSION}

In the present work, for small scale slider crank mechanism analytical kinematics studies are introduced. The studies elaborate the role of the basic design parameters on the mechanism characteristic and sensitivity. The effects of mechanism basic dimension tolerances and assembly offset errors on the mechanism quality of transmission are investigated. Relations governing the choice of the limits of these tolerances and errors according to the allowable system accuracy and uncertainty are achieved. An approach based on continuous contact mode is provided, the approach modeled the effect of the mechanism revolute joints clearances on the input-output characteristic of the mechanism. Besides that, the proposed model defines the main deviation in the mechanism performance and quality, the resultant system dead zones and hysteresis errors are concluded.

\section{REFERENCES}

[1] X. Chen and D.-W. Lee, "A Microcantilever System with Slider-Crank Actuation Mechanism", Sensors and Actuators A: Physical, vol. 226, no. Supplement C, pp. 59-68,(2015).

[2] C. Zhang and C. Rossi, "A Review of Compliant Transmission Mechanisms for Bio-Inspired Flapping-Wing Micro Air Vehicles", Bioinspiration \& biomimetics, vol. 12, no. 2, p. 025005,(2017).

[3] S. Varedi et al., "Optimal Dynamic Design of a Planar Slider-Crank Mechanism with a Joint Clearance", Mechanism and Machine Theory, vol. 86, pp. 191-200,(2015).

[4] P. Flores et al., "A Study on Dynamics of Mechanical Systems Including Joints with Clearance and Lubrication", Mechanism and Machine Theory, vol. 41, no. 3, pp. 247-261,(2006).

[5] G. Lee, BJ, Ogot, M SVi, "Dimensional Tolerance Allocation of Stochastic Dynamic Mechanical Systems through Performance and Sensitivity Analysis", Journal of Mechanical Design, vol. 115, p. 393,(1993).

[6] K.-L. Ting et al., "The Effects of Joint Clearance on Position and Orientation Deviation of Linkages and Manipulators", Mechanism and Machine Theory, vol. 35, no. 3, pp. 391-401,(2000).

[7] A. Sardashti et al., "Optimal Free-Defect Function Generation Synthesis of Four-Bar Linkage with Joint Clearance Using Pso Algorithm", Journal of Science and Engineering, vol. 1, no. 1, pp. 67-78,(2013). 
[8] J.-H. Choi et al., "Stochastic Linkage Modeling for Mechanical Error Analysis of Planar Mechanisms", Journal of Structural Mechanics, vol. 26, no. 3, pp. 257-276,(1998).

[9] S. Venanzi, "Methods for Clearance Influence Analysis in Planar and Spatial Mechanisms", PhD thesis, University of Bologna, Bologna, Italy, (2004).

[10] P. Flores, "A Methodology for Quantifying the Kinematic Position Errors Due to Manufacturing and Assembly Tolerances", Strojniški vestnik-Journal of Mechanical Engineering, vol. 57, no. 6, pp. 457-467,(2011).

[11] K.-L. Ting et al., "Output Position Uncertainty of Linkages with Prismatic Joints", in Proceedings of the 14th IFToMM World Congress, pp. 383-388, Taipei, Taiwan (2015).

[12] S. Erkaya and I. Uzmay, "Optimization of Transmission Angle for Slider-Crank Mechanism with Joint Clearances", Structural and Multidisciplinary Optimization, vol. 37, no. 5, pp. 493-508,(2009).

[13] S. Erkaya, "Effects of Balancing and Link Flexibility on Dynamics of a Planar Mechanism Having Joint Clearance", Scientia Iranica, vol. 19, no. 3, pp. 483490,(2012).

[14] V. L. Reis and K. L. Cavalca, "Comparative Analysis of the Slider Crank Mechanism with and without Contact/Friction at Revolute Joint Clearance", 22nd International Congress of Mechanical Engineering, Ribeirão Preto, SP, Brazil, (2013). 

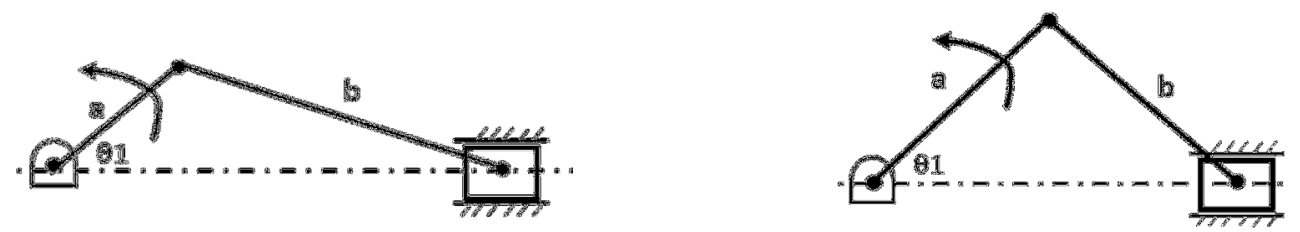

(i)

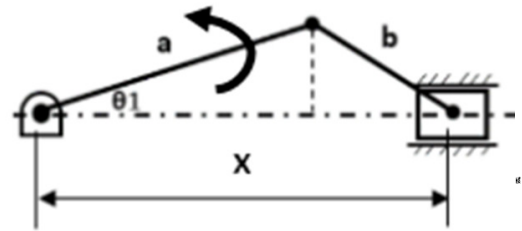

$\theta 1 \max$

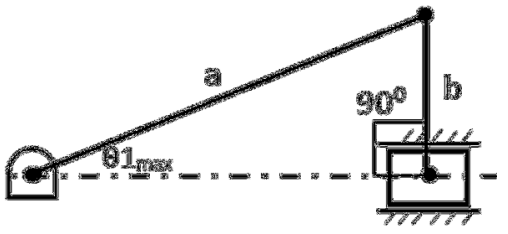

(from $\theta 1=$ zero to $\theta$ max.) c.c.w.

(ii)

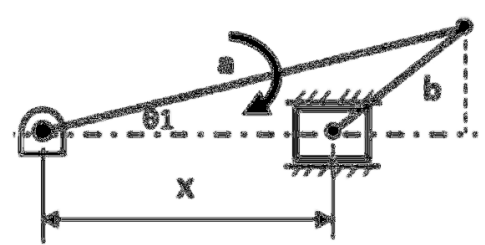

(from $\theta 1=\theta$ max. to zero) c.w.

der crank mechanism with different basic

Fig. 1. Schematic diagram of inline slider crank mechanis
dimension cases: (i) $a \leq b$, (ii) $a>b$.

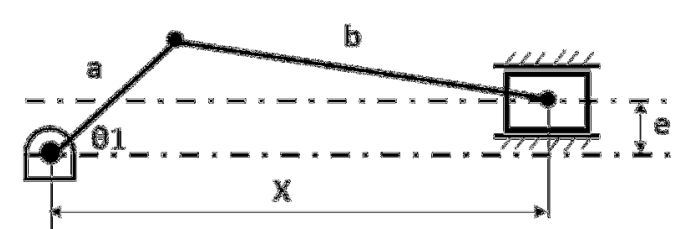

(i)

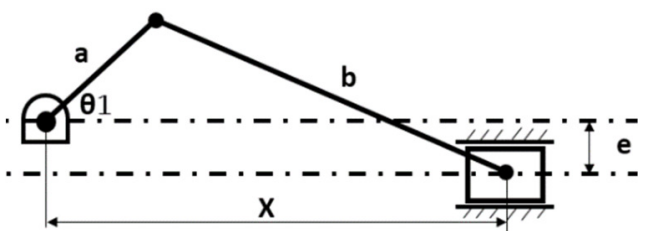

(ii)

Fig. 2. Schematic diagram of (i) Positive offset slider crank mechanism, (ii) Negative offset slider crank mechanism.

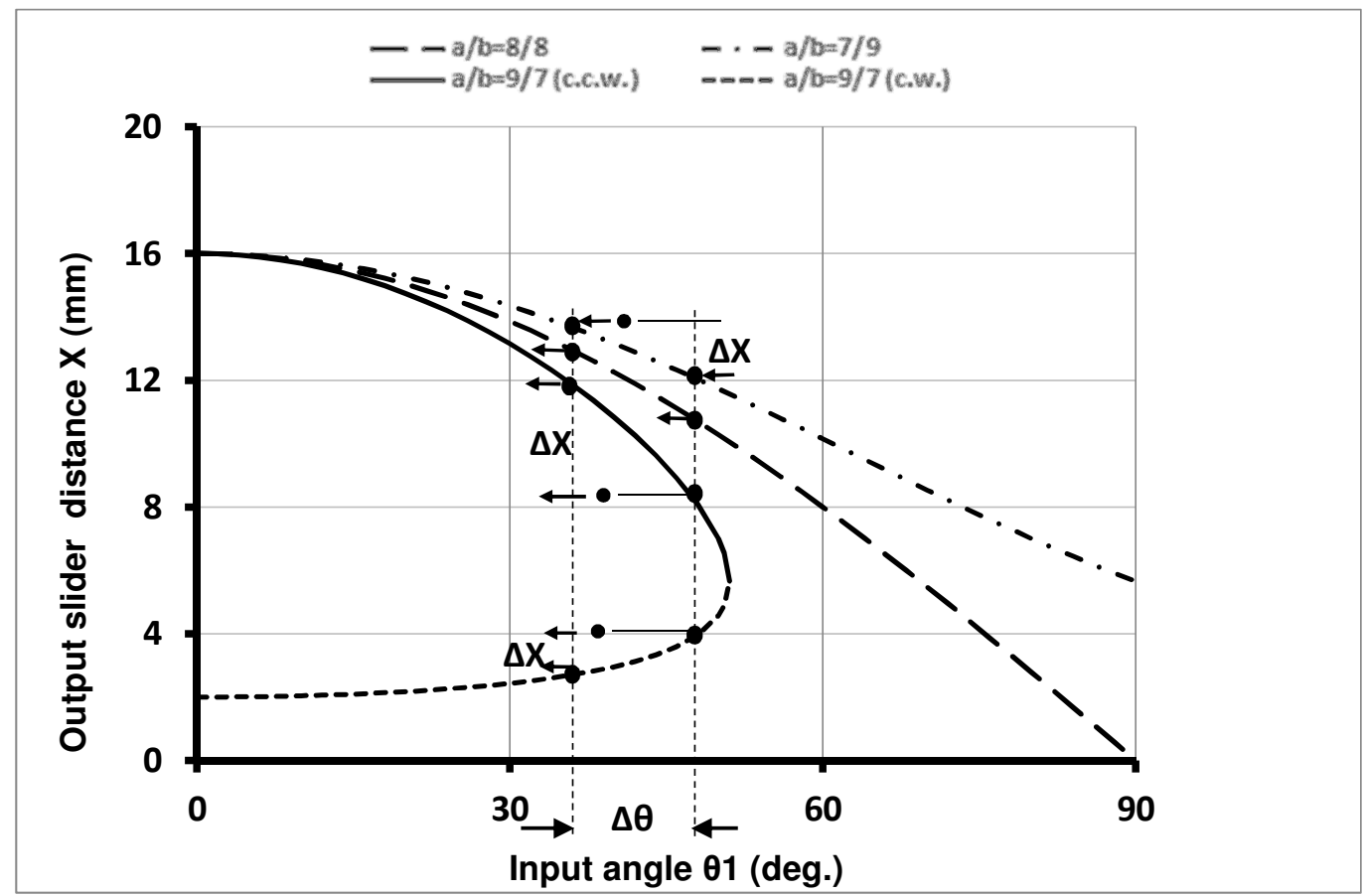

Fig. 3. Input-output relation of inline slider crank mechanism for three different basic dimensions at input angle $\theta 1$. 


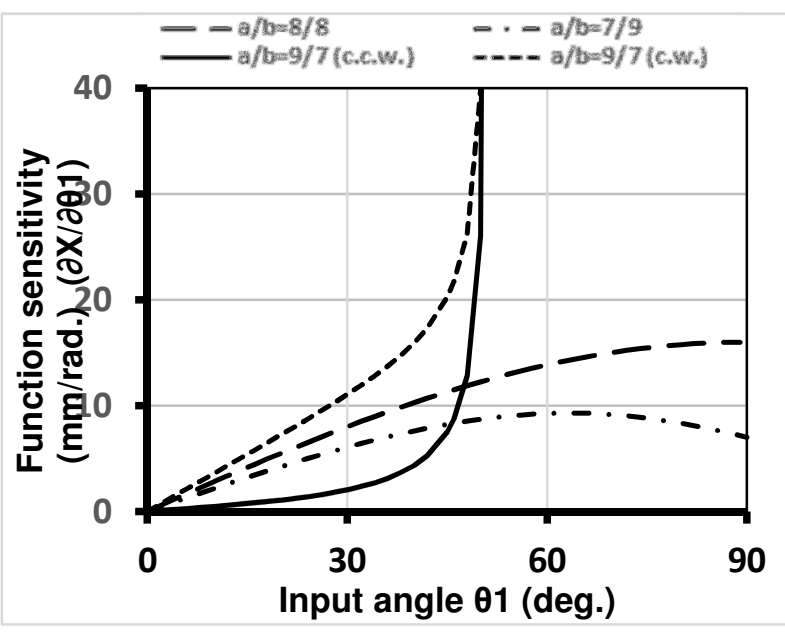

(i)

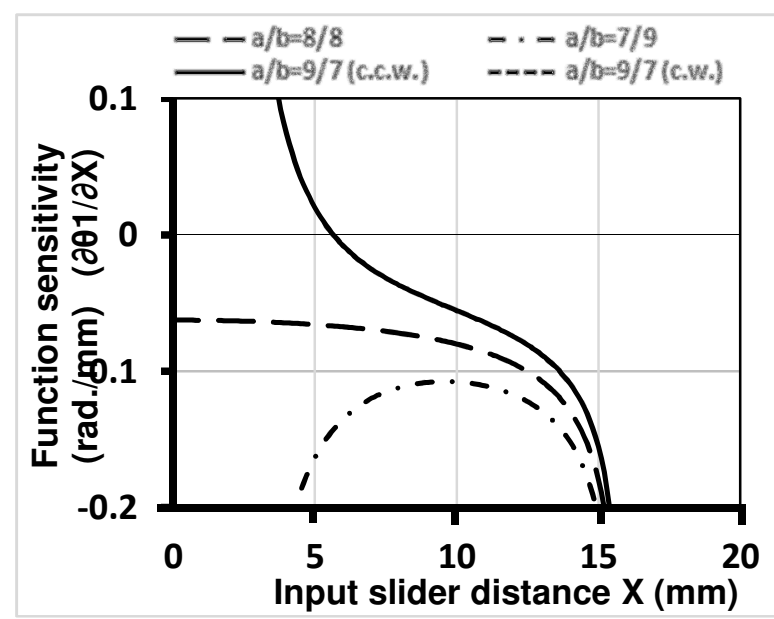

(ii)

Fig. 4. Function sensitivity for three different basic dimensions:

(i) Input angle case, (ii) Input slider distance case.

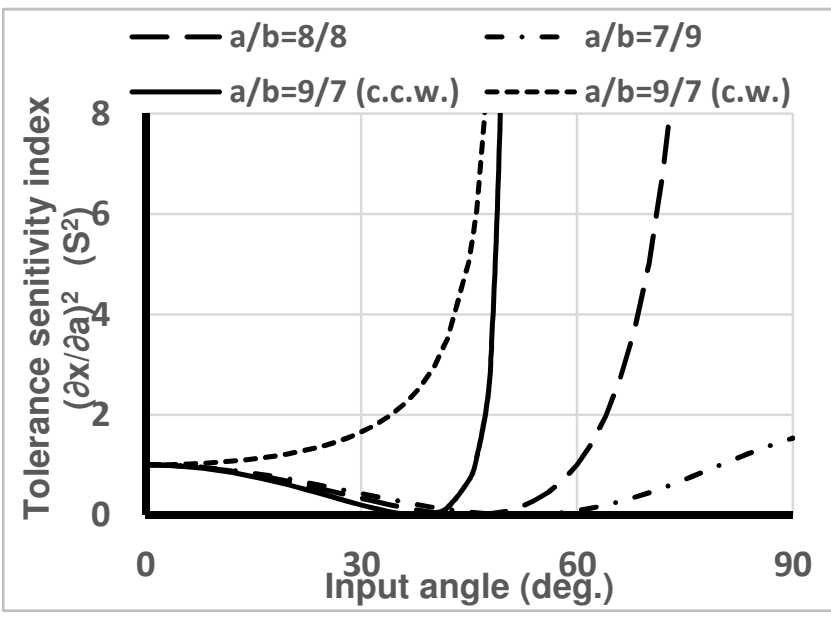

(i)

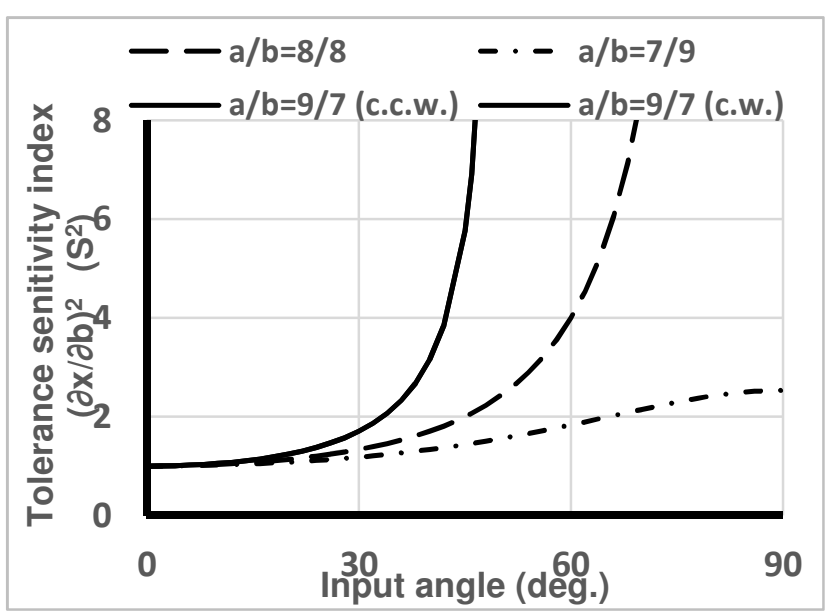

(ii)

Fig. 5. Tolerance sensitivity index $\left(S^{2}\right)$ (i) For link (a) $(\partial x / \partial a)^{2}$, (ii) For link (b) $(\partial x / \partial b)^{2}$

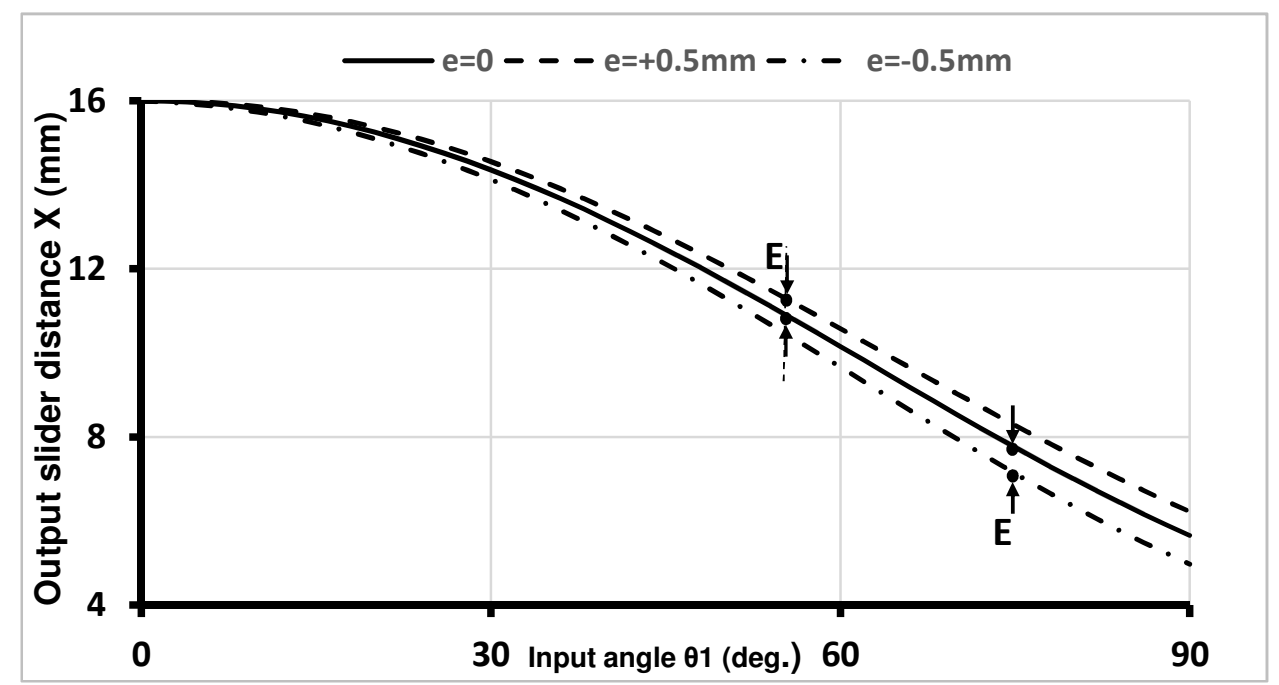

Fig. 6. Input-output relation for positive and negative offset cases at $e=0.5 \mathrm{~mm}, a=7 \mathrm{~mm}$ and $b=9 \mathrm{~mm}$. 


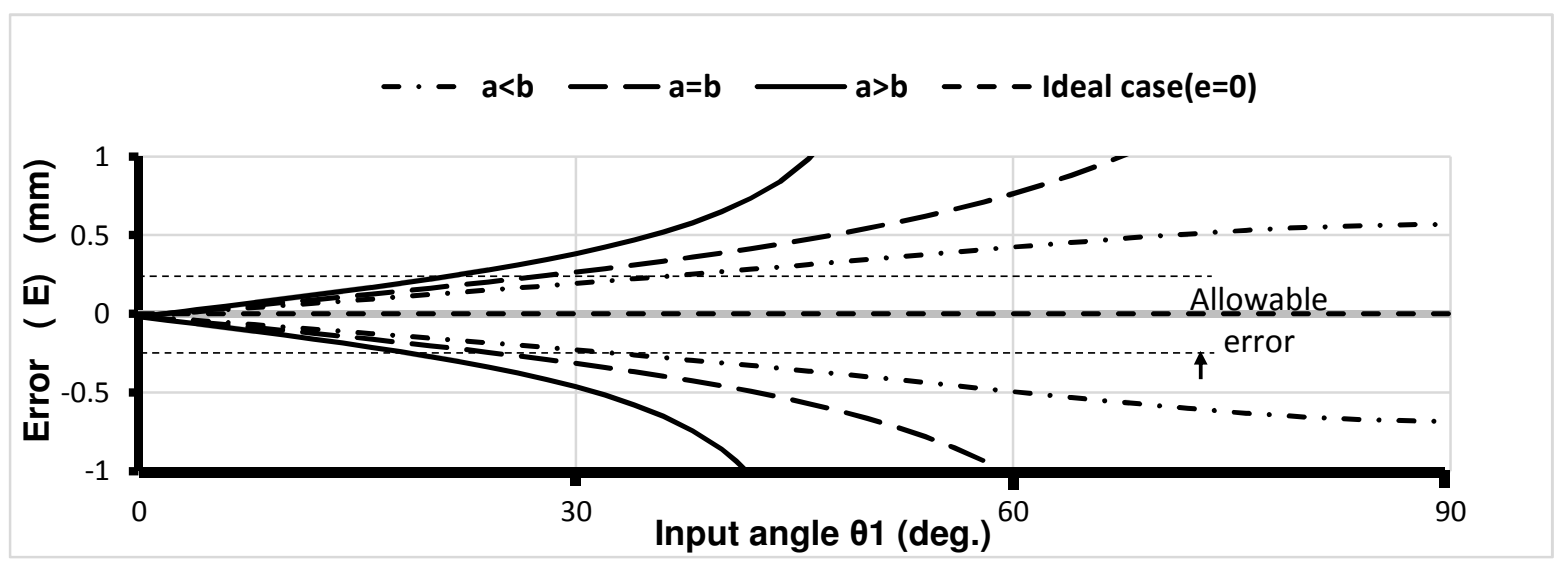

Fig. 7. Relation between the input angle and resultant error $(E)$ for positive and negative value of $e=0.5 \mathrm{~mm}$.

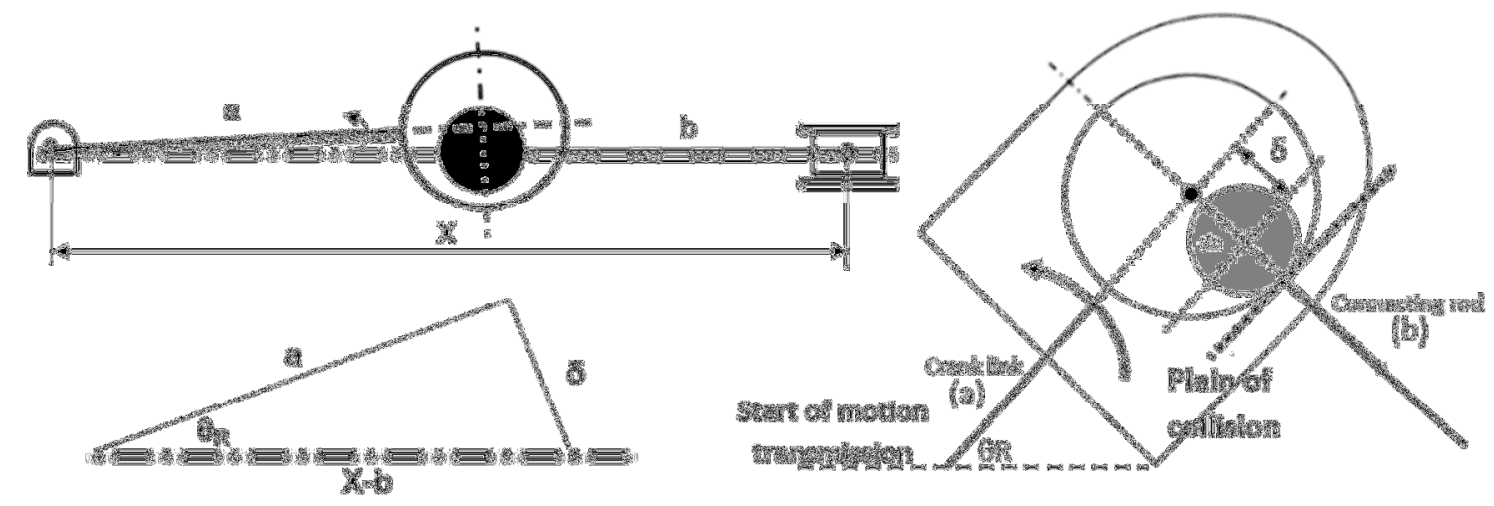

Fig. 8. Schematic representation of the slider crank and connecting rod joint.

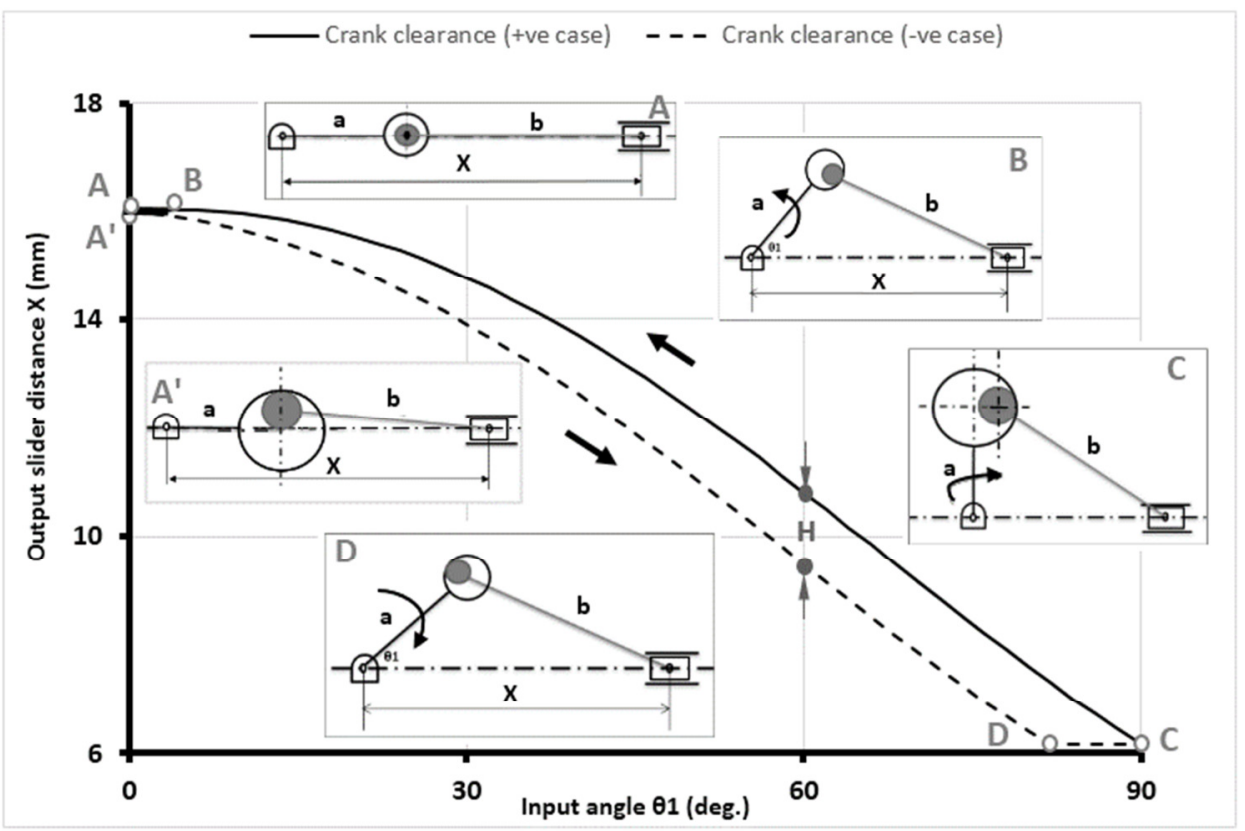

Fig. 9. Input-output relation using contact approach model for $a / b=7 / 9$ and $\delta_{c}=$ $0.5 \mathrm{~mm}$. 


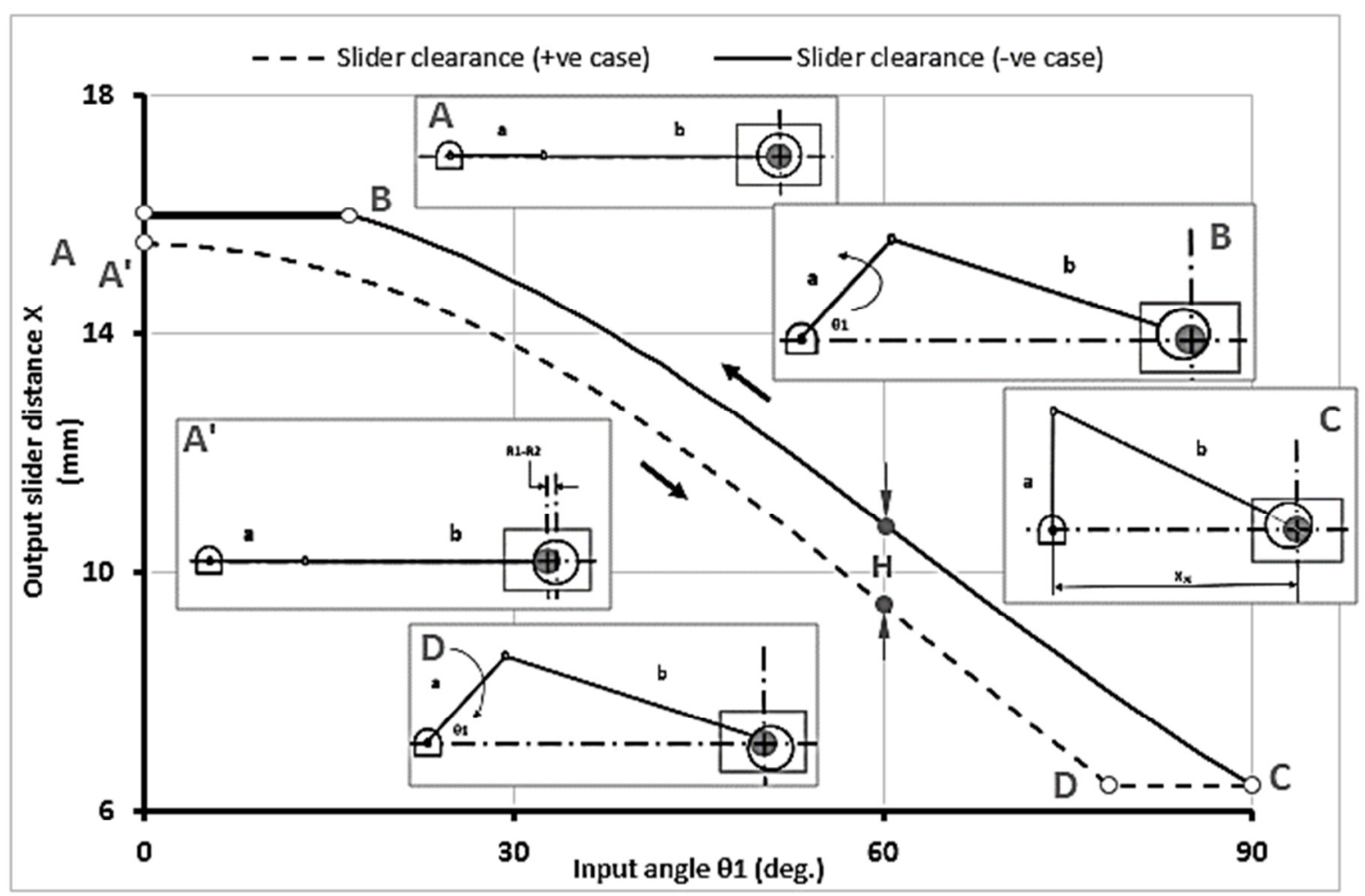

Fig.10. Input-output relation using contact approach model for $\mathrm{a} / \mathrm{b}=7 / 9$ and $\delta \mathrm{s}=$ $0.5 \mathrm{~mm}$. 